\title{
Systematic Yersinia enterocolitica in an iron overloaded and immunocompromised thalassemic patient
}

\author{
Sophia Delicou, ${ }^{1}$ Aikaterini Petrocheilou, ${ }^{2}$ Myria Pougiouka, ${ }^{2}$ Konstantinos Maragkos ${ }^{1}$ \\ ${ }^{1}$ Thalassemia and Sickle Cell Unit and ${ }^{2}$ Microbiology Laboratory, Hippokrateio General Hospital, Athens, Greece
}

\begin{abstract}
Summary
We report the case of a 43-year-old woman who presented with lower abdominal pain, maculopapular rash, arthritis of the left knee and left ankle joints, with a history of thalassemia and heavy iron overload. She was also on haemodialysis therapy three times a week for end stage renal therapy. Yersinia enterocolitica and Yersinia pseudotuberculosis cause yersiniosis, a diarrhoeal illness. Members of the genus Yersinia are gram-negative coccobacilli; they are facultative anaerobes in the family Enterobacteriaceae. Serological examinations revealed positive IgA and IgG antibodies against Yersinia enterocolitica outer membrane proteins (Yops) for YopD(4a) and Yop M(2a) and IgG for LorV (V antigen). Enteritis an reactive arthritis presented as the primary manifestation of $Y$. enterocolitica infection. Important risk factors include iron overload, cirrhosis, and immune suppression. The patient was successfully treated with oral ciprofloxacin.
\end{abstract}

\section{Introduction}

Yersinia enterocolitica belongs to the genus Yersinia, and to the family Enterobacteriacea. The genus Yersinia includes 11

Correspondence: Sophia Delicou, Thalassemia Unit, Hippokrateio Hospital, 114,Vasilisis Sofias 11527, Athens, Grecce.

Tel./Fax: +30.21320 .88000 -ext 8464

E-mail: sophiadelicou@gmail.com

Key words: Yersinia enterocolitica, iron overload, immunologically vulnerable, serologic tests.

Contributions: all authors participated in conception and design of this report and were involved in drafting and revising the manuscript; AP and MP participated in final revision of the manuscript.

Conflict of interest: the authors declare no potential conflict of interest.

Received for publication: 2 July 2017.

Revision received: 1 September 2017.

Accepted for publication: 1 September 2017.

(C) Copyright S. Delicou et al., 2017

Licensee PAGEPress, Italy

Microbiologia Medica 2017; 32:6873

doi:10.4081/mm.2017.6873

This article is distributed under the terms of the Creative Commons Attribution Noncommercial License (by-nc 4.0) which permits any noncommercial use, distribution, and reproduction in any medium, provided the original author(s) and source are credited. species, 3 of which are important human pathogens: Yersinia pestis, Yersinia enterocolitica, and Yersinia pseudotuberculosis.

Clinical presentation includes enteritis and enterocolitis, and extraintestinal manifestations such as lymphadenitis, reactive arthritis, erythema nodosum, uveitis and septicaemia. Enteric yersiniosis is a foodborne disease which is transmitted through the faecal-oral route. The species $Y$. enterocolitica is subdivided into 6 biotypes. Biotype $1 \mathrm{~A}$ is non-pathogenic while the 5 other biotypes (1B, 2-5) cause human and/or animal infections. The biotype the most frequently responsible for human infections worldwide is biotype 4, which is almost systematically associated with serotype O:3 (4/O:3), followed by bioserotype 2/O:9. Although $Y$. enterocolitica represents the third cause of bacterial diarrhoea in Europe, after campylobacteriosis and salmonellosis reports of human yersiniosis are scarce in West Africa. Overall, Y. enterocolitica infection occurs more frequently in Europe than in North America. Conditions associated with iron-overload such as chronic liver disease, hemochromatosis, and thalassemias have been associated with an increased risk of invasive yersiniosis. Yersiniosis is also a complication of iron overload syndromes treated with desferrioxamine.

\section{Case Report}

A 43-year-old female with transfusion depended thalassemia major and chronic heart and liver iron overload LIC (liver iron concentation): $17.1 \mathrm{mg} \mathrm{Fe} / \mathrm{g}$ dry tissue treated with daily s.c deferoxamine presented to outpatient clinic with a maculopapular rash over the back and the trunk. She also complained of a two-day history of lower abdominal pain, nausea, vomiting, and mild nonbloody watery diarrhoea without mucus. Her most recent blood transfusion was five days prior to the onset of symptoms. In recent past medical history she was on the 19th week of treatment with Ledipasvir-Sofosbuvir for chronic HCV infection (in a 24 weeks protocol) due to progressed cirrhosis with a liver ultrasound showing a nodular liver with splenomegaly and a transient elastography of $33.3 \mathrm{kPa}$. At the end of 4th and 12th week of treatment HCV type 1 RNA was undetectable.

On her past medical history she begun receiving haemodialysis therapy three times a week at the age of 32 years for end stage renal failure related to $\mathrm{HCV}$-cryoglobulinemic glomerulonephritis.

\section{Laboratory investigations}

Blood examination revealed: leukocytopenia WBC $3.10 \times 10^{9} / \mathrm{L}$ (neutrophils $70.0 \%$, lymphocytes $20.3 \%$, monocytes $4.7 \%$, eosinophils $1.8 \%$, basophils $0.6 \%$ ), thrombocytopenia platelet count $91 \times 10^{3} / \mathrm{uL}$ and worsening of anaemia $\mathrm{Hb} 4.9$ $\mathrm{mmol} / \mathrm{L}$ (with a baseline of $5.4 \mathrm{mmol} / \mathrm{L}$ for the patient), peripheral blood smear showed hypochromic and small hyperchromic cells 
(microspherocytes) which correlate with anisochromasia, and hypochromia. Creatinine was $0.72 \mathrm{mmol} / \mathrm{L}(0.05-0.1)$ and BUN was $40.35 \mathrm{mmol} / \mathrm{L}$ (5.36-15.36). In addition, initial laboratory investigations were significant for an elevated total bilirubin 3.60 $\mathrm{mg} / \mathrm{dL}(0.20-1.20)$ and Gamma Glutamyl Transferase $\gamma$-GT 1.72 ukat/L (0.15-0.6).Urinalysis revealed mild haematuria and 2.5 grams of proteinuria. C-reactive protein was $121 \mathrm{ug} / \mathrm{L}(0-50)$. Peripheral blood cultures were drawn, and urine and stool was sent for culture. Serologic tests for Coxsackievirus A B, Echovirus, Enterovirus and Yersinia were sent. Abdominal ultrasonography and plain computed tomography scanning showed massive splenomegaly and significant mesenteric lymphadenitis. The patient was treated supportively without antibiotics. Two days later she returned for scheduled transfusion visit. She was afebrile complained for central abdominal pain and frequent bowel movements without blood; she also presented arthritis of the left knee and left ankle joints. The maculopapular rash subsided. The laboratory findings at that time were as follows: haemoglobin was 4.53 $\mathrm{mmol} / \mathrm{L}$. White blood count was $2.3 \times 10^{9} / \mathrm{L}$ (neutrophils $80.0 \%$, lymphocytes $30.3 \%$, monocytes $3.7 \%$, eosinophils $2.8 \%$, basophils $0.6 \%$ ), and C-reactive protein was $241 \mathrm{ug} / \mathrm{L}$. Liver and renal function tests did not differ from the previous time. All subsequent blood cultures were negative, as were stool studies. Serological examinations revealed positive $\operatorname{IgA}$ and $\operatorname{IgG}$ antibodies against Yersinia enterocolitica outer membrane proteins (Yops) for YopD(4a) and Yop M(2a) and IgG for LorV (V antigen). Oral ciprofloxacin $500 \mathrm{mg}$ twice daily was administered for 10 days.

Yersinia antibodies IgA and IgG were positive and stayed positive within 3 and 6 months after the infection with no recurrent or persistent illnesses, or symptoms including pharyngitis, lymphadenopathy, fever, headaches, arthralgia, and diarrhoea. Twelve months after infection only IgG antibodies remained positive (Table 1).

\section{Discussion and Conclusions}

Y. enterocolitica and $Y$. pseudotuberculosis cause yersiniosis, a diarrheal illness; human infection with $Y$. enterocolitica is much more common than human infection with Y. pseudotuberculosis. Sporadic yersiniosis has been observed worldwide. Overall, $Y$. enterocolitica infection occurs more frequently in Europe than in North America; it is rarely observed in tropical countries (6). The organism was first described by Schleifstein and Coleman in 1939 as... an unidentified microorganism resembling Bacterium lignieri and Pasteurella pseudotuberculosis, and pathogenic for man. $Y$. enterocolitica transmission occurs mainly through food, especially pork products. Yersinia enterocolitica and Yersinia pseudotuberculosis are important foodborne pathogens that cause infections through contaminated refrigerated food. Their cold tolerance mechanisms are therefore of special interest. Adaptation to cold involves changes in protein synthesis and in cell membranes to overcome diminished transcriptional and translational efficiency and reduced fluidity of cell membranes. Other sources include untreated surface water and blood transfusions, the latter because the organism proliferates in iron-rich environments at refrigerator temperatures $(5,6,10)$.

The incubation period for yersiniosis is typically 4 to 6 days (range 1 to 14 days). The onset of Yersinia gastroenteritis can be more subacute than other diarrheal pathogens. Clinical manifestations of acute yersiniosis include diarrhoea, abdominal pain, and fever; nausea and vomiting may also occur (10). Erythema nodosum associated with Yersinia enterocolitica is not rare (9). Reactive arthritis typically affects the large weight-bearing joints and begins several weeks after the onset of acute infection.

Patients with beta-thalassemia are more susceptible to bacterial infections for a variety of reasons, including absence of the spleen. Infection is a major cause of morbidity in thalassemic patients and is second only to hemosiderin cardiomyopathy as a cause of mortality. Cherchi GB reported $10 \%$ of thalassemic patients in one centre had $Y$. enterocolitica infection diagnosed over a 1-year period.

In our case hypesplenism affects cellular and humoral immunity especially in combination with cirrhosis. Patients with homozygous beta-thalassemia $(2,7,8)$ are at increased risk for serious Yersinia enterocolitica infections. Data have suggested that Yersinia may be a ferrophilic bacterium that requires a higher level of readily available iron for the initiation of growth than do other pathogens.

Yersinia does not elaborate a siderophore but can use those from other bacteria. In the gastrointestinal tract there is an abundance of siderophore compounds from other organisms, creating an environment favourable for yersinial growth. In rare cases, $Y$. enterocolitica septicaemia has been observed following transfusion with packed red cells $(2,7,8)$. Yersinia organisms are ferrophilic and capable of multiplying slowly in stored units of red cells at cold temperatures before they are transfused. In one prospective study conducted in 1998 to 2000, the incidence of transfusion associated Yersinia sepsis was 1 in 23.7 million red cell transfusions.

The relation between virulent YE infection, iron, and deferoxamine is peculiar and has been clarified recently. YE lacks a natural high-affinity iron chelator and therefore cannot absorb iron directly, it is dependent on bacterial siderophores in the gut, for

Table 1. Serum antibodies of Yersinia.

\begin{tabular}{|c|c|c|c|c|c|c|c|c|c|c|}
\hline & \multicolumn{4}{|c|}{ Clinical diagnosis } & \multicolumn{6}{|c|}{ Without clinical manifestations } \\
\hline & \multicolumn{2}{|c|}{ Diagnosis } & \multicolumn{2}{|c|}{10 days with ciprofloxacin } & \multicolumn{2}{|c|}{1 month } & \multicolumn{2}{|c|}{3 months } & \multicolumn{2}{|c|}{12 months } \\
\hline & IgA & IgG & $\operatorname{Ig} \mathrm{A}$ & $\operatorname{Ig} G$ & $\operatorname{Ig} A$ & $\operatorname{Ig} G$ & $\operatorname{Ig} A$ & $\operatorname{Ig} G$ & $\operatorname{Ig} A$ & $\operatorname{Ig} \mathbf{G}$ \\
\hline YopM(2a) & Positive & Positive & Positive & Positive & Positive & Positive & Positive & Positive & Negative & positive \\
\hline YopH(2b) & Negative & Negative & Negative & Negative & Negative & Negative & Negative & Negative & Negative & Negative \\
\hline LorV & Negative & Negative & Negative & Positive & Negative & Positive & Negative & Positive & Negative & Positive \\
\hline YopD(4a) & Positive & Positive & Positive & Positive & Positive & Positive & Positive & Positive & Negative & Positive \\
\hline YopN(4b) & Negative & Negative & Negative & Negative & Negative & Negative & Negative & Negative & Negative & Negative \\
\hline YopP(30) & Negative & Negative & Negative & Negative & Negative & Negative & Negative & Negative & Negative & Negative \\
\hline YopE(5) & Negative & Negative & Negative & Negative & Negative & Negative & Negative & Negative & Negative & Negative \\
\hline
\end{tabular}


which it does have high-affinity receptors. Transfusion may increase tissue iron available for invasive YE, and the presence of deferoxamine, a siderophore obtained from Streptomyces pilosus, further enhances the virulence of YE by providing additional siderophores in large quantities for which abundant iron is already available. The combination of an iatrogenic siderophore and abundant iron markedly enhances the virulence of YE.

Desferrioxamine has long been administered parenterally as an iron chelating agent to patients with iron overload, however, it adds to the risk of infection by $Y$. enterocolitica. Our patient was iron overloaded on desferrioxamine therapy $(7,8)$ at the time of the acute infection. Treatment for HCV and haemodialysis rendered our patient further immunologically vulnerable.

The organism, often cultured in blood, can also be recovered from stool, appendix, mesenteric lymph nodes, and distal ileum. Infections of extraintestinal sites include lymphadenitis, arthritis, osteomyelitis, pneumonia, endocarditis, meningitis, and dermatitis.

The most common post-infectious sequelae are erythema nodosum and reactive arthritis; these are particularly common in Northern Europe and, for the latter, among those with the HLAB27 tissue type.

Yersinia is a gram-negative bacillus in the Enterobacteriacae family $(6,10)$. Yersinia can appear small and coccobacillary in Gram-stained smears. It exhibits bipolar staining described as a safety pin shape on Giemsa staining. Yersinia grow on blood, chocolate, and MacConkey agar, but may be overgrown by other organisms due to slow growth. Yersinia can form pinpoint colonies on both blood agar and MacConkey agar in 24 hours, particularly Y. pseudotuberculosis. Yersinia are catalase positive, oxidase negative and ferment glucose. Y. enterocolitica appears as small, lactose-negative colonies on MacConkey in 48 hours. Serologic tests can be used to support a diagnosis of yersiniosis. They are serogrouped using antisera produced against cell surface lipopolysaccharide antigens, known classically as the $\mathrm{O}$ antigens. For $Y$. enterocolitica, biogroup and serotype are correlated. The most common are serotype O:9 biotype 2, serotype O:3 biotype 4 and serotype O:8 biotype 1B. Simple agglutination assays have been developed for diagnosis of yersiniosis. In addition, enzyme linked immunosorbent assays (ELISA) and immunoblotting can be used to detect IgG, IgA, and IgM class antibodies. A positive IgM assay supports the diagnosis of acute yersiniosis, as does a fourfold rise in antibody titers between acute and convalescent titers drawn several weeks apart.

Antibody levels begin to rise within the first week of illness, peak the second week and then return to normal within 3-6 months. In our case positive antibodies followed acute infection and persisted for many months. Antibodies develop against the Yersinia outer membrane proteins (Yops). It has been reported that the assays used to detect antibodies against Yops are more sensitive and specific than stool culture and other serologic diagnostic methods. In our case serologic findings were interpreted positive with post-infectious sequelae oscillating antibody titers upon the activity of their illness.

Lately a multiplex PCR assay with dual priming oligonucleotide system (DPO system-based mPCR) was developed for the simultaneous detection of Yersinia enterocolitica.
The mortality rate associated to $Y$. enterocolitica infection can reach as high as 50\% in immunocompromised individuals. Antimicrobial treatment (1) varies among serogroups, and the microorganism is usually susceptible in vitro to cotrimoxazole, aminoglycosides, tetracycline, and fluoroquinolones, but is resistant to penicillin, ampicillin and first-generation cephalosporins due to the presence of two chromosomal genes encoding beta-lactamase, blaA and blaB, which confer a broad-spectrum or firstgeneration cephalosporins resistance, respectively.

Recommendations arising from this case report indicate that diarrhoea, even in the absence of fever and abdominal findings, in a patient with iron overload should alert the clinician to the possibility of yersinia infection. For patients with $b$ thalassemia, especially if symptoms occur shortly following a blood transfusion, clinical suspicion should be heightened.

\section{References}

1. Baumgartner A, Küffer M, Suter D, et al. Antimicrobial resistance of Yersinia enterocolitica strains from human patients, pigs and retail pork in Switzerland. Int J Food Microbiol 2007;115:110-4.

2. Fàbrega A, Vila J. Yersinia enterocolitica: pathogenesis, virulence and antimicrobial resistance. Enferm Infecc Microbiol Clin 2012;30:24-32.

3. Hansen MG, Pearl G, Levy M. Intussusception due to yersinia enterocolitica enterocolitis in a patient with $\beta$-thalassemia. Arch Pathol Lab Med 2001;125:1486-8.

4. Hoelen DW, Tjan DH, Schouten MA, et al. Severe yersinia enterocolitica sepsis after blood transfusion. Neth J Med 2007;65:301-3.

5. Neubauer H, Sprague LD, Scholz H, Hensel A. [Diagnosis o Yersinia enterocolitica infections: a review on classical identification techniques and new molecular biological methods]. Berl Munch Tierarztl Wochenschr 2001;114:1-7. [Article in German].

6. Rahman A, Bonny TS, Stonsaovapak S, Ananchaipattana C. Yersinia enterocolitica: epidemiological studies and outbreaks. J Pathog 2011;2011:239391.

7. Roussos A, Stambori M, Aggelis P, et al. Transfusion-mediated Yersinia enterocolitica septicemia in an adult patient with betathalassemia. Scand J Infect Dis 2001;33:859-60.

8. Schubert S, Autenrieth IB. Conjugation of hydroxyethyl starch to desferrioxamine (DFO) modulates the dual role of DFO in Yersinia enterocolitica infection. Clin Diagn Lab Immunol 2000;7:457-62.

9. Yotsu R, Mii S, Hayashi R, et al. Erythema nodosum associated with Yersinia enterocolitica infection. J Dermatol 2010;37:819-22.

10. Zheng H, Sun Y, Lin S, et al. Yersinia enterocolitica infection in diarrheal patients. Eur J Clin Microbiol Infect Dis 2008;27:741-52. 